\title{
O VIVER DO PORTADOR CHAGÁSICO CRÔNICO: possibilidades de ações do enfermeiro para uma vida saudável
}

\author{
Angélica Porto de OLIVEIRAa, Luciane Feira GOMES \\ Sidnéia T essmer CASA RINc, Hedi Crecencia H eckler de SI QU EI RAd
}

\section{RESUM 0}

O bjetivou-se conhecer como vivem os portadores chagásicos crônicos em dois municípios da região sul do Rio G rande do Sul, traçando possíveis ações do enfermeiro para uma vida mais saudável. 0 estudo foi de caráter descritivo e exploratório com uma abordagem qualitativa. Os dados foram coletados nos domićlios de dez clientes portadores crônicos da D oença de Chagas através de entrevista semiestruturada. Os resultados mostraram que a sintomatologia da doença não se manifestou na fase aguda, sendo que a maioria dos sujeitos faz uso de medicações paliativas para alívio dos sintomas, existindo grandes limitações no desenvolvimento das atividades cotidianas, principal mente nos portadores da forma cardíaca. Reitera-se o valor da equipe de saúde, em especial do enfermeiro, no manejo clínico, pois quando iniciado precocemente, propicia uma melhor qualidade de vida e uma elevação da expectativa de sobrevivência.

D escritores: D oença de Chagas. Assistência à saúde. Saúde pública. E stilo de vida. E nfer magem em saúde comunitária.

\section{RESUMEN}

E I objetivo fue conocer cómo viven los portadores chagásicos crónicos en dos municipios de la región sur de R io G rande do Sul, B rasil, cómo enfrentan esa patología y trazar posibles acciones del enfermero para una vida más saludable E I estudio fue de carácter descriptivo y exploratorio con abordaje cualitativo. L os datos fueron colectados en los domicilios de diez clientes portadores crónicos de la enfermedad de Chagas a través de entrevista semiestructurada. $L$ os resultados evidenciaron que la sintomatología de la enfermedad no se manifestó en la fase aguda siendo que la mayoría de los sujetos usa medicaciones paliativas para el alivio de los síntomas, existiendo grandes limitaciones en el desar rollo de las actividades cotidianas, sobre todo, en los portadores de la forma cardíaca. Se reitera el valor del equipo de salud, en especial, del enfermero, en el manejo clínico de estos clientes, pues cuando se inicia precozmente, puede propiciar una mejor calidad de vida y resultar en la elevación de la expectativa de su supervivencia.

D escriptores: E nfermedad deChagas. P restación deatención desalud. Salud pública. E stilo devida. E nfermería en salud comunitaria.

Título: E I vivir del portador chagásico crónico: posibilidades de acciones del enfermero para una vida saludable

\section{ABST RACT}

This study, carried out in two cities in southern R io G rande do Sul, B razil, aims to getunderstand how people with chronic Chagas disease live in order to draw possible nursing actions towards a healthier life T he study has a descriptive and exploratory character with a qualitative approach. D ata w ere collected through semi-sctructured interviews carried out with ten Chagas disease chronic car riers at their houses. The results showed that the sickness symptomatology did not appear in the acute phase of the disease; it also showed that most of the carriers make use of palliative medication to alleviate symptoms. There are great limitations to their performing daily chores, especially for heart-affected carriers. T he support of the health team, especially the nurse's, is valuable to the clinical management of the patient. W hen it is started in the early stages of the disease, a better quality of life and a longer life expectancy can be reached.

Descriptors: Chagas disease. D elivery of health care. P ublic health. L ife style Community health nursing.

T itle: T he life of the patient with chronic Chagas disease: possible nursing actions tow ards a healthy life

a Enfermeira da Estratégia de Saúde da Família de Piratini, M embro do Grupo de Estudo e Pesquisa Gerenciamento E cossistêmico em Enfer magem/ Saúde (GEES), Piratini, Rio Grande do Sul, Brasil.

b Enfermeira, Bolsista do Programa de Iniciação Científica (PIC) da Educacional Anhanguera no ano de 2008, Pinheiro M achado, Rio Grande do Sul, Brasil.

c M estre em Enfermagem, Enfer meira da Secretaria M unicipal de Saúde de Piratini, M embro do GEES, Piratini, Rio G rande do Sul, Brasil.

d D outora em E nfermagem, D ocente do Programa de Pós-G raduação em E nfer magem - Curso de M estrado e D outorado - da U niversidade

Federal do Rio Grande (FURG), Líder do GEES, Rio Grande, Rio Grande do Sul, Brasil. 


\section{INT RODUÇÃO}

A tripanossomíase americana, ou D oença de Chagas, como ficou conhecida, descrita em 1909 por Carlos Chagas, tem como agente etiológico 0 Trypanossoma cruzi, um protozoário flagelado intracelular ${ }^{(1)}$. Essa patologia apresenta grande distribuição no continente americano, compreendendo desde o sul dos Estados U nidos até a Argentina. Estudos têm mostrado que existem 16 a 18 miIhões de pessoas infectadas, ocorrendo 23 mil mortes por ano $0^{(1,2)}$.

A principal forma de transmissão é a vetorial, através das fezes dos triatomíneos, também conhecidos como "barbeiros" ou "chupões". A transmissão pode ocorrer por intermédio de transfusão sanguínea ou via placentária, durante a gestação ${ }^{(1,3)}$. Pode ocorrer, também, a transmissão acidental em laboratório ou pelo leite materno, sendo que estas são de pouca significância epidemiológica ${ }^{(1,3)}$. Recentemente, também foram relatados casos da D oença de Chagas na forma aguda, consequentes à transmissão por via oral, após ingestão de caldo de cana contaminado com fezes de barbeiros ${ }^{(3)}$.

Como características, a doença em sua fase aguda apresenta sinais e sintomas quase sempre inespecíficos, porém em seu curso clínico crônico pode gerar comprometimento car díaco, provocando cardiomegalia ou patologias digestivas, megacólon e/ ou megaesôfago ${ }^{(1,4)}$.

As manifestações resultantes das afecções chagásicas podem interferir na satisfação das necessidades básicas dos portadores crônicos, diminuindo, significativamente, a sua qualidade de vida. Dessa forma, pode haver necessidade constante de cuidados especial izados de saúde ${ }^{(3,5)}$. Assim, o enfermeiro ao assistir o portador de Chagas crônico, precisa incorporar no seu plano assistencial o levantamento das necessidades objetivas e subjetivas dessa clientela, com a finalidade de proceder à programação do atendimento de cada um conforme suas particularidades individuais ${ }^{(6,7)}$. As necessidades subjetivas envolvem os aspectos relacionados ao que a pessoa sente e ao que pensa de sua vida, enquanto outros abarcam um conjunto de elementos de ordem objetiva, porém ambas precisam ser atendidas para a satisfação das necessidades ${ }^{(6)}$.

0 trabalho em saúde é col etivo, executado por vários agentes, dentre eles o profissional enfermeiro. Cada um desses agentes é possuidor de conhecimentos e saberes específicos de sua área, os quais são utilizados como instrumentos ${ }^{(8)}$. N essa perspectiva, os profissionais de saúde encontram um campo de atuação com base na assistência/ cuidado, buscando entender o portador da D oença de Chagas. Essa assistência/ cuidado precisa, antes de qualquer ação interventiva, conhecer esse ser humano que vive de maneira particular, porque se encontra infectado pelo parasita chagásico.

0 conhecimento a respeito do viver particular, desse grupo de sujeitos, pode levar à construção de subsídios capazes de traçar estratégias específicas para um cuidado de enfermagem mais direcionado e com maior eficiência. Portanto, este estudo possibilita o crescimento do conhecimento/ saber da enfermagem, assim como auxilia no avanço da prática profissional a respeito desse grupo específico.

Essa questão significativa em torno do viver dos clientes chagásicos levou a traçar o seguinte objetivo de estudo: conhecer como vivem os portadores chagásicos crônicos de dois municípios do extremo sul do Rio Grande do Sul e delinear possíveis ações do enfermeiro para uma vida mais saudável dessa população.

\section{MÉTODOS}

T rata-se de um estudo de caráter descritivo e exploratório com uma abordagem qualitativa(9), realizado nos meses de fevereiro a dezembro de 2008.

0 palco do estudo consta de dois municípios (A e B ) da 3a Coordenadoria Regional de Saúde do Rio Grande do Sul, com população entre 12.939 (M unicípio A) e 20.225 habitantes (M unicípio B)(10). E sses locais possuem grande extensão rural etêm caráter endêmico da D oença de Chagas.

O levantamento dos portadores crônicos de Chagas, residentes na área de abrangência, foi real izado por meio dos cadastros de usuários existentes na Estratégia de Saúde da Família (ESF), nos respectivos municípios. A ssim, identificaramse vinte e dois portadores crônicos da Doença de Chagas, sendo que quinze residiam no M unicípio A e sete, no M unicípio B. A partir da identificação, decidiu-se escolher aleatoriamente, através do método do sorteio, cinco pessoas de cada município, total izando assim, os 10 sujeitos do estudo. Justifica-se o número reduzido de sujeitos neste estudo, uma vez que na pesquisa qualitativa há o in- 
teresse do pesquisador em conhecer bem o objeto de estudo, possibilitando-Ihe maior aprofundamento e abrangência na sua compreensão(9).

Aos sujeitos da pesquisa foi garantido o direito de desistir em qualquer momento do estudo, assim como o anonimato. Para isto, foram identificados com a letra S, inicial da palavra sujeito, seguida do número decimal, conforme a sequência das entrevistas realizadas (de S1 até S10). Todos os preceitos éticos que envolvem pesquisa com seres humanos ${ }^{(11)}$ foram respeitados, sendo que 0 estudo foi aprovado mediante parecer 024/2008, Ata 67/ 2008, pelo Comitê de Ética em Pesquisa da Santa Casa de M isericórdia de Pelotas, Rio Grande do Sul.

Para a coleta de dados, foi utilizada a entrevista semiestruturada, e os dados capturados foram transcritos na íntegra, organizados e mapeados, para uma melhor visualização da sua totalidade $^{(9)}$. Para a análise dos dados, procedeu-se a leitura flutuante com a finalidade de impregnação do seu conteúdo e encontrar as unidades de registro, para formar os temas ${ }^{(9)}$. Os temas constituíram-se nos seguintes tópicos: caracterização dos sujeitos, conhecimento do diagnóstico, enfrentamento da doença e a possibilidade de ações do enfermeiro na qualidade de vida do cliente chagásico.

\section{RESULTADOS E DISCUSSÃO}

\section{Caracterização dos sujeitos}

A maioria dos entrevistados é do sexo masculino e tem entre 51 e 82 anos, com idade média de 63 anos. A renda familiar mínima dos sujeitos do estudo foi de $R \$ 190,00$, e a máxima de $R \$$ 500,00 , portanto uma renda média mensal de $R \$$ 289,00 . A lém disso, cinco dos sujeitos entrevistados são aposentados.

Sabe-se que a aposentadoria precoce em portadores da D oença de Chagas, muitas vezes, ocorre pelo comprometimento da saúde decorrente das complicações tardias da doença, no qual é necessário tratamento constante e cuidados especiais. Entretanto, o valor que recebe, geral mente, não é suficiente para garantir as despesas com o tratamento e o essencial para a sua subsistência. N esse sentido, a situação financeira dos sujeitos do estudo interfere na questão social e psicológica, já que os coloca à margem do desenvolvimento normal da sociedade. Assim, entende-se que a
D oença de Chagas é tanto de natureza biológica como psicológica e social, já que afeta a população mais pobre ${ }^{(12)}$, a qual não possui os recursos financeiros indispensáveis para enfrentar as adversidades que a patologia impõe e, desse modo, de forma sistêmica, encontra-se atingida como um todo.

Todos os clientes entrevistados, em algum momento de suas vidas, residiram na área rural, em casas de adobe e/ ou palha, porém, atualmente, apenas dois dos sujeitos do estudo continuam residindo nessas regiões todos possuem casas de alvenaria.

A migração do trabalhador rural para as cidades é um fenômeno conhecido como "êxodo rural", no qual os indivíduos buscam nas cidades meIhores condições de vida. A través dos relatos dos sujeitos deste estudo, todos os entrevistados já haviam residido, anteriormente, na área rural e conheciam o vetor da D oença de Chagas, contato com o inseto em algum momento na vida. A migração rural-urbana do cliente chagásico é apontada na literatura e conhecida como a urbanização da doença que, até então, era uma particularidade do meio rural, porém as condições socioeconômicas encontradas nas cidades são tão precárias quanto aquelas vivenciadas no seu meio de origem $^{(13)}$.

Ao serem questionados quanto ao conhecimento em relação ao vetor "barbeiro", todos referiram que conheciam o inseto, e que fora na infância o contato que tiveram com o mesmo. Porém, um dos sujeitos também mencionou que ainda hoje continua tendo contato com o inseto.

[...] já vi aqui em casa, diversas vezes, na cidade e no interior, quando achou o barbeiro mato (S2).

[...] na casa que eu morava na infância existia o barbei ro, lembro que caía do teto da casa eque picavam os meus pés e de meus irmãos (S5).

[...] era nova ainda fui picada no seio pelo barbeiro, anos e anos fiquei complicada [ ...] (S6).

[...] onde morava, o chupão era que nem mosquito tinha muito, mas na casa atual não tem chupão [ ...] (S10).

0 vetor da doença multiplica-se em casas rudimentares, ditas de "pau-a-pique" ou conhecidas como de adobe, as quais são moradias apropriadas para os insetos e não, para pessoas ${ }^{(14)}$. A 
execução do combate ao vetor, juntamente com a melhoria do panorama agrário diminuíram as impropriedades habitacionais, resultando na erradicação do barbeiro em muitos locais do Brasil(14). E sse fato foi observado neste estudo, no qual todos os sujeitos relataram ter residido em casas de adobe, porém hoje, com a melhoria habitacional, residem em casas de alvenaria, mesmo os que continuam morando na zona rural.

Ainda em relação à afirmação de $\$ 2$, sobre a existência do vetor nos domicílios, a literatura consultada aponta para o fato de que, no Rio Grande do Sul, há registros de 11 espécies de T riatomineae: T riatoma infestans, T riatoma sordida, T riatoma rubrovaria, Triatoma circummaculata, T riatoma oliveirai, T riatoma klugi, T riatoma carvalloi, T riatoma plantesis, T riatoma del pontei, Panstrong ylus tupynambai e o Panstrongylus megistus. Os dois primeiros apresentam hábitos sinantrópicos, sendo que os demais, hábitos silvestres ${ }^{(15)}$.

Contudo, a literatura consultada chama a atenção para 0 fato de que o Panstrogylus megistus pode habitar as residências humanas, quando seus nichos em matas são destruídos e suas fontes de alimentação escasseiam por alterações ambientais. T ambém, o T riatoma rubrovaria, mesmo apresentando hábitos silvestres, tem sido capturado em domicílios. Esse fato comprova características de adaptação desse inseto aos ambientes antrópicos e serve de preocupação e alerta para a necessidade de se manter vigilância entomológica eficiente, com a finalidade de detectar mudanças de comportamento desses insetos, os quais invadem e estabelecem suas colônias nas moradias humanas, el evando o risco de transmissão do T ripanossoma cruzi(15).

\section{Conhecimento do diagnóstico}

E m relação ao conhecimento do diagnóstico, os sujeitos do estudo referiram que tiveram a doença detectada em situações rotineiras, como: a realização de exames para controle da hipertensão arterial, ou em busca de respostas para sintomas inespecíficos, como constipação intestinal, cansaço, falta de ar, aceler ação dos batimentos cardíacos, hipertensão arterial, conforme falas a seguir:

F ui ao banco de sangue para fazer uma doação para o pai de uma conhecida, lá me disseram que tinha um probleminha no sangue que tinha sido descoberto na outra doação do sangue que fiz três anos antes desse dia. E ntão, me passaram para o médico de lá mesmo que me disse que tinha dado no exame que eu tinha Chagas [...] (S2).

[ ...] comecei a sentir falta de ar e cansaço por qualquer caminhadinha que dava, também sentia meu coração acelerar, foi aí que fui ao médico, e el e disse que achava que eu tinha coração crescido (S3).

H á mais ou menos cinco anos, quando subiu a pressão, e procurei atendimento médico, aí o médico solicitou exames para Chagas (S6).

F ui ao médico, porque tinha intestino parado, aí ele pediu exame e deu positivo (S7).

A literatura consultada menciona que a maioria dos portadores da forma crônica de Chagas fica sabendo da sua condição tardiamente, ou porque apresentam algum sintoma, ou ainda ao procurarem algum serviço de saúde descobrem, por acaso, que estão infectados ${ }^{(3,4,16)}$. N este estudo, este fato também foi confirmado, uma vez que todos os entrevistados procuraram os serviços de saúde tardiamente, ou seja, quando já apresentavam algum sintoma da doença crônica, tais como: realização de exames para controle da hipertensão arterial ou na busca de respostas para sintomas inespecíficos, dentre eles a constipação intestinal, cansaço aos mínimos esforços, falta de ar e aceleração dos batimentos cardíacos.

Entretanto, três dos sujeitos do estudo descobriram que eram portadores crônicos, ao realizarem doação de sangue, e um entrevistado solicitou exame sorológico para Doença de Chagas ao médico, porque tinha casos da doença na família.

$\mathrm{N}$ este contexto, a doença de Chagas, geralmente, passa imperceptível na fase aguda e quando o portador toma conhecimento que se encontra infectado pelo parasita, a doença já está na fase crônica. A literatura consultada ratifica esses achados ao afirmar que a fase aguda tem sinais e sintomas quase sempre inespecíficos e que podem se apresentar de forma inaparente ${ }^{(3,17)}$. A ponta, também, para o fato de que o paciente, ao ultrapassar a fase inicial da doença, passa por um Iongo período assintomático (de 10 a 30 anos), sem evidências de complicações, podendo até mesmo apresentar exames el etrocardiográficos e radiológicos de coração, esôfago e cólon sem alterações ${ }^{(4)}$. 
E $m$ relação à forma de manifestação da doença, seis clientes mencionaram serem portadores da forma cardíaca; dois da forma intestinal; um cliente, da forma mista e ainda um, com diagnóstico indeter minado. Todos os clientes referiram al gum tipo de dor ou desconforto refer ente à patologia e apenas um não faz tratamento contínuo.

A patologia crônica é descrita na literatura por apresentar, além da forma indeterminada, formas cardíaca, digestiva, mista e congênita ${ }^{(18)}$.

E mbora não se possa negar que, nos últimos cem anos, houve muitas conquistas em relação ao controle da Doença de Chagas. Estima-se que, a cada ano, no Brasil, cerca de seis mil pacientes irão morrer por causas relacionadas à doença, fazendo com que a mesma continue sendo consider ada como um importante problema da saúde pública brasileira, na medida em que existem cerca de três e meio milhões de pacientes crônicos remanescentes, dos quais $20 \%$ a $30 \%$ apresentam comprometimento cardíaco(13).

\section{Enfrentamento da doença e a possibilidade de ações do enfermeiro na qualidade de vida do cliente chagásico}

Ao analisar como os portadores enfrentam a doença, percebe-se que a maioria faz uso de medicações paliativas, ou seja, tratam os sintomas e complicações da doença através de uso contínuo de remédios. E ssa real idade confirma-se, pois, até o momento, não há cura para a for ma crônica da doença, sendo que esses clientes não se beneficiam clinicamente do tratamento oferecido aos acometidos da forma aguda, visto que essa ter apêutica tem a finalidade de suprimir a parasitemia e não, tratar as patologias decorrentes da ação do tripanosso$m a^{(3,19)}$.

Aos portadores crônicos, resta apenas receber o tratamento sintomático, na tentativa de minimizar os sintomas provenientes da cronificação da doença.

Os principais sintomas da forma cardíaca são pal pitações, dor precordial, dispnéia, entre outros; enquanto na forma digestiva são: disfagia, regurgitação, epigastral gia, dinofagia, entre outros(3). Essas manifestações repercutem, expressivamente, no estilo de vida dos portadores, uma vez que essas dores e desconfortos diminuem a produtividade dos indivíduos e alteram o equilíbrio dos componentes que geram bem-estar.
Desse modo, influenciam nos aspectos biológico, social, psicológico e espiritual do cliente chagásico, interferindo na sua qualidade de vida e bem-estar de forma sistêmica, ou seja, cada uma das dimensões influencia é influenciada de maneira interdependente.

Alguns autores mostram que a forma cardíaca de Chagas é de maior restrição ao doente chagásico, sendo a principal causa de óbito. Entretanto, a forma digestiva traz alterações significativas ao longo do tubo digestório, alterando a mobilidade e a morfologia desse sistema ${ }^{(3,4,19)}$. Os entrevistados acometidos, principalmente pela forma cardíaca, possuem limitações no desenvolvimento de suas atividades cotidianas, o que vem interferir não apenas no seu bem-estar, mas também na questão do trabalho, dificultando o ganho necessário para sua sobrevivência.

Os dados evidenciam que todos os sujeitos, de uma ou outra forma, sentem dor e/ ou desconforto nos mais diversos segmentos do seu corpo. E ssas manifestações que se apresentam de maneira, mais ou menos constante, ao longo do dia, fazem que suas atividades cotidianas fiquem reduzidas e, assim, elas influenciam no seu bem-estar, conforme exemplificado nas falas a seguir:

T enho problema na garganta que me faz engasgar com facilidade(S2).

[ ...] sinto cansaço físico, aperto no peito, às vezes sinto dificuldade para engolir e me engasgo, fico tonto quando faço algum esforço e, às veze, fico com a barriga muito inchada depois de me alimentar, esses sintomas vêm piorando de uns meses pra cá (S3).

D or no corpo (S5).

T udo faz mal, sempre sofrendo do intestino (S6).

D or de cabeça ena coluna [ ...] D or na coluna, no estômago de alguma coisa que come (S8).

Sempre doente, com dor por tudo (S9).

Quanto ao tratamento medicamentoso empregado de forma contínua, os sujeitos aludiram não se utilizarem de nenhum tipo de tratamento específico para a patologia, porém oito dos entrevistados fazem tratamento para al gum tipo de sintomatologia e fizeram referência quanto ao uso de medicações anti-hipertensivas, antidiabéticas e para 
controle dos níveis lipêmicos. Ainda, mencionaram utilizar alguma medicação para controle da cardiopatia e para 0 alivio dos sintomas intestinais provocados pela doença, conforme segue:

Sim, faço tratamento para pressão (S1).

Sim, para pressão e coração (S3).

Sim, AAS (S4).

Sim, diurético e sinvastatina (S5).

F aço, para o coraçãao, intestino e pressão alta (S6).

Para Chagas não, só para pressão e diabetes (S10).

U m dos entrevistados mencionou que a doença não interfere em suas atividades diárias, contudo relata utilizar-se de medicação para contornar o desconforto gerado pela sintomatologia da doença.

$\mathrm{N}$ ão atrapalha de fazer nada, para o intestino parado é usado óleo (S7).

Q uanto ao enfrentamento da doença e a influência na qual idade de vida, os entrevistados apresentam déficit no desenvolvimento das atividades diárias, como no trabalho doméstico ou no trabaIho assalariado.

[ ...] não consigo fazer nada que fazia antes, porque tudo me cansa muito e me dá um aper to no peito que me sufoca! Se não tivesse a doença, eu estaria trabaIhando, tive que parar por causa disso (S3).

[ ...] hoje não consigo trabalhar da mesma forma que antes, dá uma cansei ra, fico sem forças etenho que descansar (S10).

Sim nas atividades de casa. P or horas, não consigo fazer as coisas por causa da dor, fico indisposta (S6).

Este estudo revelou que todos os sujeitos vivem o seu cotidiano e enfrentam a doença de forma singular. Entretanto, ao associar os desconfortos como fator intrínseco à qualidade de vida de qualquer ser humano, pode-se assegurar que a Doença de Chagas abala o equilíbrio do estilo de vida dos portadores(20) que necessitam de cuidados específicos, para minimizar os efeitos negativos dessa patologia.
OIhando o cliente chagásico neste contexto, um tratamento bem conduzido e iniciado precocemente, aumenta a sobrevida dos clientes e, consequentemente, sua qualidade de vida. Além disso, permite a prática das atividades habituais, desde que as mesmas não impliquem esforços físicos ao se tratar da forma cardíaca ${ }^{(3,5,6,19)}$.

E ssas considerações vêm demonstrar a importância da equipe de saúde, em especial do enfermeiro, no manejo clínico do cliente chagásico, particularmente das formas cardíacas, pois quando bem conduzido e iniciado precocemente, pode resultar na el evação da expectativa de sobrevivência dessa clientela.

É nessa perspectiva que se entende que o enfermeiro tem a possibilidade de exercer a função assistencial das pessoas portadores da Doença de Chagas. E ntende-se que o cuidado sistemático do enfermeiro a esses clientes, oferecendo orientações fundamentadas no conhecimento da patologia, é capaz de amenizar os efeitos adversos do cliente chagásico crônico. Além disso, ele pode fazer a busca ativa nas áreas endêmicas, visando com isso, a atender todos os infectados e oportunizar-Ihes, precocemente, atendimento adequado.

$\mathrm{N}$ esse sentido, considera-se a possibilidade de delinear ao enfermeiro, ações capazes de melhorar 0 estilo de vida dessas pessoas. Cabe a esse profissional, como educador da saúde, incentivar os portadores chagásicos a manter hábitos saudáveis, para conseguir um melhor equilíbrio no seu bem-estar. I gualmente, ao controlar de forma constante os desconfortos ocasionados pela doença, o enfermeiro pode oportunizar um estar melhor a essa clientela.

\section{CONSIDERAÇÕES FINAIS}

0 estudo realizado, utilizando o método de entrevista semiestruturada para coleta de dados, junto aos portadores de doença crônica de Chagas, permitiu envolver a questão pesquisa e alcançar os objetivos propostos.

A pesquisa mostrou-se relevante, não apenas pela temática que se caracteriza como um grave problema sócio-político-cultural e envolve questões de saúde coletiva, mas principalmente, porque permitiu conhecer como vivem os portadores chagásicos crônicos, como enfrentam a sintomatologia dessa patologia e, assim, discutir o delineamento de possíveis ações do enfermeiro para uma vida mais saudável dessa população. 
A experiência vivenciada durante a realização dessa pesquisa contribuiu para o aumento significativo do conhecimento dessa temática e, especialmente, na formação das pesquisadoras, não apenas como futuras profissionais, mas repercutiu, sobretudo, no ser pessoa humana e cidadã. A o dialogar com os portadores da Doença de Chagas, foi possível vivenciar fatos concretos e acompanhar nos seus domicílios os dilemas, as dificuldades que vivenciam.

Entretanto, o fator de grande relevância neste aspecto de aprendizagem foi, sem dúvida, o ensinamento que cada um dos sujeitos conseguiu passar com sua vivência e a forma utilizada no enfrentamento dessa patologia.

Esse é um aprendizado que não se obtém e não se desenvolve em encontros teóricos na academia. Cada domicílio visitado foi uma verdadeira recepção que, de forma organizada, manifestava a fraternidade, o prazer em viver, a luta para conseguir a cura e a vontade de ser mais saudável. Esses sentimentos fizeram aflorar reflexões profundas nas pesquisadoras e influenciaram, positivamente, na experiência vivida para continuar com outras temáticas a buscar entender os problemas que afetam a população.

Ressalta-se a importância das ações do enfermeiro em conscientizar os clientes, através da educação em saúde, principalmente das regiões endêmicas da Doença de Chagas, a manter um estilo de vida saudável. Essas ações do enfermeiro podem ajudá-los no enfrentamento da doença, protegê-los dos desconfortos resultantes dessa patologia e, assim, melhorar o estilo de vida e aumentar suas expectativa e qualidade de vida.

E ntende-se que um estilo de vida saudável deve incluir a promoção e prevenção da saúde, abarcando hábitos saudáveis quanto à alimentação, controle de peso, lazer, exercícios físicos, abster-se de substâncias nocivas, principalmente fumo e álcool. Acredita-se que essa pode ser mais uma das formas, utilizadas pelo enfermeiro, capaz de produzir resultados positivos para o doente chagásico crônico, pois assim, é possível que o paciente ele sintase mais útil e, dessa forma, consiga despertar para um viver melhor, mais saudável.

\section{REFERÊNCIAS}

1 Kieling C, M achado ARL. D oença de Chagas. In: Duncan BB, Schimdt M I, G iugliani, ERJ. M edicina ambulatorial: condutas de atenção primária baseadas em evidencias. 3a ed. Porto A legre: A rtmed; 2004. p. 1482-4.

2 Vinhaes M C, D ias JCP. D oença de Chagas no Brasil. Cad Saúde Pública [ I nternet] . 2000 [ citado 2009 jul 14] ;16(2):7-12. D isponível em: http:/ / www.scielosp. org/ pdf/ csp/ v16s2/ 3480.pdf.

3 M inistério da Saúde (BR), Secretaria de Vigilância em Saúde. Guia de vigilância epidemiológica. 6ạ ed. Brasília (D F ); 2005.

4 Sanchez-L ermen R LP, D ick E, Salas J A P, F ontes CJ F. Sintomas do trato digestivo superior e distúrbios motores do esôfago em pacientes portadores da forma indeter minada da doença de Chagas crônica. Rev Soc Bras M ed Trop [I nternet]. 2007 [ citado 2009 jul 14] ;40(2):197-203. D isponível em: http:/ / www.sciel o. $\mathrm{br} / \mathrm{pdf} / \mathrm{rsbmt} / \mathrm{v} 40 \mathrm{n} 2 /$ a10v40n2.pdf.

5 Porto CC. Vademecum de clínica médica. 2a ed. Rio de Janeiro: G uanabara Koogan; 2007.

6 Oliveira AP, Gomes LF, Siqueira HCH. Portadores da doença de Chagas: sua vida e assistência à saúde. ANUIC. 2008;11(12):753.

7 F undação O sval do Cruz. D oença de Chagas [ I nternet] . Rio de Janeiro; 2008 [ citado 2008 jun 14] . D isponível em: http:// www.fiocruz.br/chagas/cgi/ cgilua.exe/ sys/ start.htm?sid=1.

8 Silva JB, Kirschbaum DIR, Oliveira I. Significado atribuído pelo enfermeiro ao cuidado prestado à criança doente crônica hospitalizada acompanhada de familiar. Rev Gaúcha Enferm [ Internet] . 2007 [ citado 2009 nov 07];28(2):250-9. Disponível em: http:/ / www.seer.ufrgs.br/ index.php/R evistaG auchade Enfermagem/article/ view/ 3176/ 1749.

9 M inayo M CS. 0 desafio do conhecimento: pesquisa qualitativa em saúde. 10a ed. São Paulo: Hucitec; 2007

10 M inistério do Planejamento, Orçamento e G estão (BR), Instituto Brasileiro de G eografia e E statística. Cidades [I nternet]. Rio de Janeiro; 2009 [ citado 2009 nov 07] . Disponível em: http:// www.ibge. gov.br/ cidadesat/ topwindow.htm?1.

11 M inistério da Saúde (BR), Conselho Nacional de Saúde. Resolução 196, de 10 de outubro de 1996: diretrizes e normas regulamentadoras de pesquisa envolvendo seres humanos [ Internet]. Brasília (DF); 1996 [ citado 2007 ago 10] . D isponível em: http:/ / 
conselho.saude.gov.br/ docs/ Resolucoes/ Reso196. doc.

12 M agnani C, Oliveira BG, G ontijo ED. M itos e comportamentos do paciente submetido ao implante de marcapasso na doença de Chagas. Cad Saúde Pública [ I nternet] . 2007 [ citado 2009 jul 14] ;23(7):162432. Disponível em: http:/ / www.scielo.br/ pdf/csp/ v23n7/ 13.pdf.

13 Oliveira J R. A tenção integral ao paciente chagásico: uma proposta para o cuidar. Arq Bras Cardiol [ I nter net] . 2005 [ citado 2009 jul 14] ;84(1). D isponível em: http:/ / www.scielo.br/ pdf/ abc/ v84n1/ 22996.pdf.

14 A mato N eto V, Pasternak J. Centenário da doença de Chagas. Rev Saúde Pública [I nter net] . 2009 [ citado 2009 jul 14];43(2):381-2. Disponível em: http:/ / www.scielosp.org/ pdf/ rsp/ v43n2/ CE.pdf.
15 Bedin C, M ello F, W ilhelms T S, Torres M A, Estima $C$, Ferreira CF, et al. Vigilância ambiental: doença de Chagas no Rio Grande do Sul. Bol Epidemiol [ I nternet] . 2009 [ citado 2010 jul 30] ;11(3):1-8. Disponível em: http:/ / www.saude.rs.gov.br/dados/ 1265820158577BE \%20V 11-N 3-IN T ERN ET .pdf.

16 Costa E M A, Carbone M H. Saúde da família: uma abordagem interdisciplinar. Rio de Janeiro: Rubio; 2004.

17 A guiar ZN, Ribeiro M CS. Vigilância e controle das doenças transmissíveis. 2aed. São Paulo: M artinari; 2006.

19 M inistério da Saúde (BR ). D oenças infecciosas e parasitárias: guia de bolso. 6aed. Brasília (DF ); 2006.

20 Siqueira-Batista R, Gomes AP. Infections by Trypanosoma cruzi: revisiting Chagas deseases. J Bras M ed. 2002;82(5):28-41.

\section{Endereço da autora / Dirección del autor /} Author's address:

Angélica Porto de Oliveira

Rua D r. L uís de Oliveira L essa, 36

96490-000, Piratini, RS

E-mail: angelport083@ hotmail.com
Recebido em: 29/ 06/ 2010

A provado em: 23/ 08/ 2010 